\title{
The Impacts of Degassing on Space Charge Characteristics And DC Conductivity in Semicon- Bonded XLPE For HVDC Cable Applications
}

\author{
M. Hao, A. Fazal, A. S. Vaughan, G. Chen \\ Tony Davis High Voltage Laboratory \\ University of Southampton \\ Southampton, SO17 1BJ, UK \\ M.Hao@soton.ac.uk
}

\author{
Y. Zhou, C. Zhang \\ Smart Grid Research Institute \\ Beijing, China
}

\begin{abstract}
High voltage direct current (HVDC) cables are one of the key components for the HVDC power transmission system. HVDC cables for higher voltage level, larger power capacity and higher reliability are still desired for the power industry as part of a global power strategy. One of the major concerns related to HVDC applications is the presence of space charge within the dielectrics, which distorts the electric field distribution and contributes to accelerated ageing and consequent failure of the cable insulation. The byproducts generated from the crosslinking process during the manufacture of XLPE insulation for HVDC cable are one of the major sources of hetero-charge formation in dielectrics. Therefore, degassing is widely applied in cable manufacture for the removal of such crosslinking byproducts. In this paper, composite XLPE samples were produced that include two layers of semiconductive polymer (semicon), which are thermally bonded on the top and bottom of the XLPE insulation, to mimic a real cable manufacturing process. The samples were degassed under vacuum and elevated temperature for 1, 3 and 6 days and their space charge characteristics and conductivities were then measured at an applied field of $40 \mathrm{kV} / \mathrm{mm}$. The influence of the degassing period and the semicon material formulation on space charge formation and distribution in the system are discussed and the findings are compared with undegassed samples.
\end{abstract}

Keywords - Space charge; PEA; XLPE; HVDC cable; semiconducting material; charge injection; conductivity

\section{INTRODUCTION}

In the literature, it has been long recognized that the thermal and mechanical properties of PE can be significantly enhanced by the crosslinking [1] and, for this reason, XLPE cables are often the best choice in the power industry for HVDC applications. Crosslinking is the process whereby the polymer structure is modified through the formation of chemical bonds between polymer chains and the most common method of chemical crosslinking is to trigger a thermally activated catalyst, such as, dicumyl peroxide (DCP), by subjecting the polymer to high temperatures. However, there are drawbacks associated with crosslinking; for DCP, every decomposed peroxide molecules gives byproducts, which are contained within the XLPE [2]. These byproducts can act as impurities and significantly affect the dielectric properties of the insulation, such as the formation of space charge [1]. Space charge in insulating materials is known to affect electrical conduction and can distort the electric field distribution in the system, thereby accelerating breakdown [3]. There are reports that crosslinking residues can act as localized charge trapping centers [4], giving a rise to space charge in the insulating material $[5,6]$. The most common treatment to improve the performance of cables is degassing, in which process, the XLPE is subjecting to a moderately high temperature in vacuum, thereby, removing volatile residual constituents. Over the past decade, various studies have reported on space charge formation within as received as well as degassed XLPE. However, limited attention has been given to the effect of the length of the degassing period on subsequent HVDC performance. The work reported here describes the impact of the degassing period on the space charge behavior of composite XLPE samples, where the XLPE insulation is sandwiched between two layers of semiconductive polymer (semicon). The aim was to simulate the structure of a power cable, in which the insulation layer is similarly located between two semicon layers. The influence of the semicon material formulation on space charge formation and its distribution is discussed and the findings are compared with undegassed XLPE samples.

\section{EXPERIMENTAL}

\section{A. Materials}

The samples used in this study were produced from a cablegrade low density polyethylene (LDPE) and the semicon electrodes were made from the same grade of LDPE loaded with carbon black. Both materials were supplied by the Smart Grid Research Institute (China). The crosslinking agent was dicumyl peroxide (DCP), supplied by Sigma-Aldrich.

\section{B. Sample Preparation}

The LDPE and DCP $(2 \%)$ were first mixed in bulk at $120^{\circ} \mathrm{C}$ for $15 \mathrm{~min}$ in a Thermo PolyLab mixer. Sheet samples of the resulting LDPE containing DCP ( $250 \mu \mathrm{m}$ thickness) and the semicon ( $\sim 50 \mu \mathrm{m}$ thickness) were separately prepared by hot pressing at a temperature below the activation threshold of the crosslinking agent. The semicon electrodes were then hot pressed (double sided) into the plaque sample at $200{ }^{\circ} \mathrm{C}$ for 10 min to ensure crosslinking and good interfacial bonding, 
schematically illustrated in Fig. 1. The resultant thickness of the composite samples was approximately $250 \mu \mathrm{m}$ (including 200 $\mu \mathrm{m}$ XLPE and $50 \mu \mathrm{m}$ semicon). Melting of the LDPE prior to crosslinking is key in sample processing and, therefore, to ensure good bonding, both materials were first allowed to melt for $2 \mathrm{~min}$ at $120^{\circ} \mathrm{C}$. The applied load was maintained during the cooling process and the sample was removed from the hot press once $120{ }^{\circ} \mathrm{C}$ reached and, finally, was cooled down to room temperature.

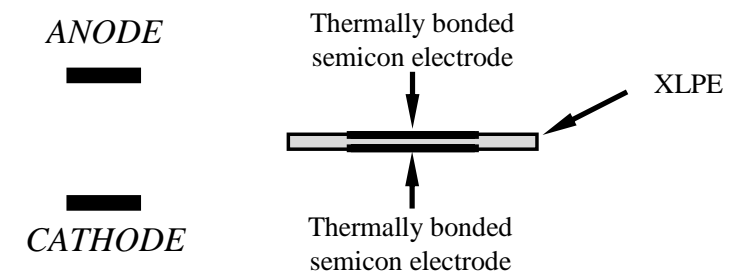

Fig. 1. Schematic illustration of the semicon electrode setups used for PEA and conductivity measurements.

\section{Degassing}

A standard XLPE treatment adopted by cable manufacturers is degassing, in which XLPE is subjected to a moderately high temperature under vacuum to remove volatile residual byproducts. In this work, degassing of semicon-bonded XLPE samples was carried out in a vacuum oven at a constant temperature of $80{ }^{\circ} \mathrm{C}$. The samples were then cooled down to room temperature and sealed in polyethylene bags, prior to tests. The degassing durations of 1,3 and 6 days were used, with the aim of expelling crosslinking byproducts and to highlight any influence this may have on the material performance.

\section{Sample Characterization}

Space charge evolution was measured using the PEA technique. For this, the sample was inserted between the lower and upper electrodes and DC electric field of $40 \mathrm{kV} / \mathrm{mm}$ was applied. Space charge data were acquired both with the field applied to the sample and during its subsequent decay, with the sample short circuited. All PEA tests were carried out at room temperature, and the evolution of charge, polarization (voltage on) and depolarization (voltage off) for each stressing period lasted $60 \mathrm{~min}$. The calibration for PEA tests was done by applying a low voltage $(2 \mathrm{kV})$ for a short period of time $(5 \mathrm{~s})$ to show the capacitive charges on both electrodes. This low voltage was chosen to ensure no space charge developed in the bulk sample.

For electrical conduction, the sample was placed in a test cell containing opposing $25 \mathrm{~mm}$ diameter electrodes. A constant DC voltage of $8 \mathrm{kV}(40 \mathrm{kV} / \mathrm{mm})$ was applied and the resulting current was recorded for $3 \mathrm{~h}$ at room temperature.

\section{RESULTS AND DISCUSSION}

Fig. 2 shows DC conductivity plots of degassed and undegassed XLPE sample. In this work, the conductivity of 3hour measurement is regarded as the steady state conductivity, as the difference between this value and the very long period result ( 3 days) is less than 5\%. The result of Fig. 2 clearly highlights the conductivity of undegassed samples is significantly higher than that of degassed sample. This could be due to the byproducts generated from the crosslinking reaction, such as acetophenone, $\alpha$-methylstyrene, cumyl alcohol and water [7]. It has been previously reported the acetophenone leads to the formation of ionic charges in XLPE when it coexists with water and, therefore, leads to increased electrical conductivity [8]. This suggests that undegassed samples (with more byproducts) would contain more free charge carriers in comparison with degassed XLPE. Similar findings were reported elsewhere [9], in which the authors claimed that the byproducts in undegassed XLPE sample were responsible for the increased in conductivity. .

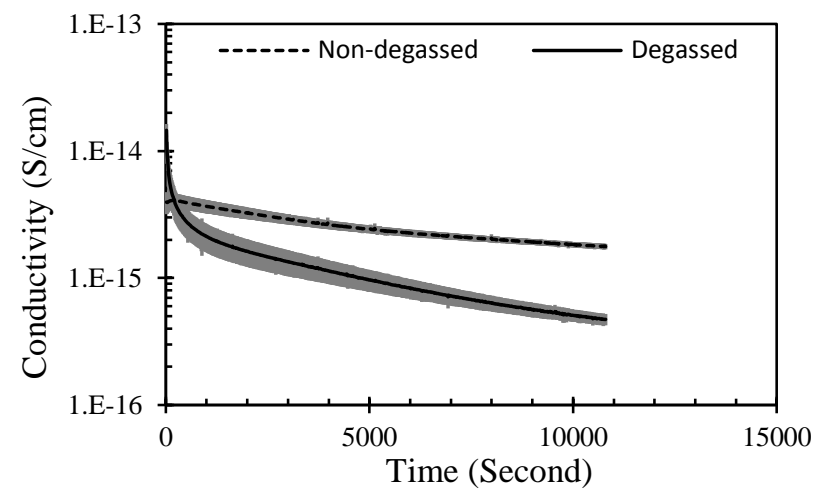

Fig. 2. DC conductivity plots of XLPE thermally bonded with Sc electrodes. The applied voltage was $40 \mathrm{kV} / \mathrm{mm}$ at room temperature. Note, the standard error of the mean highlighted in grey color.
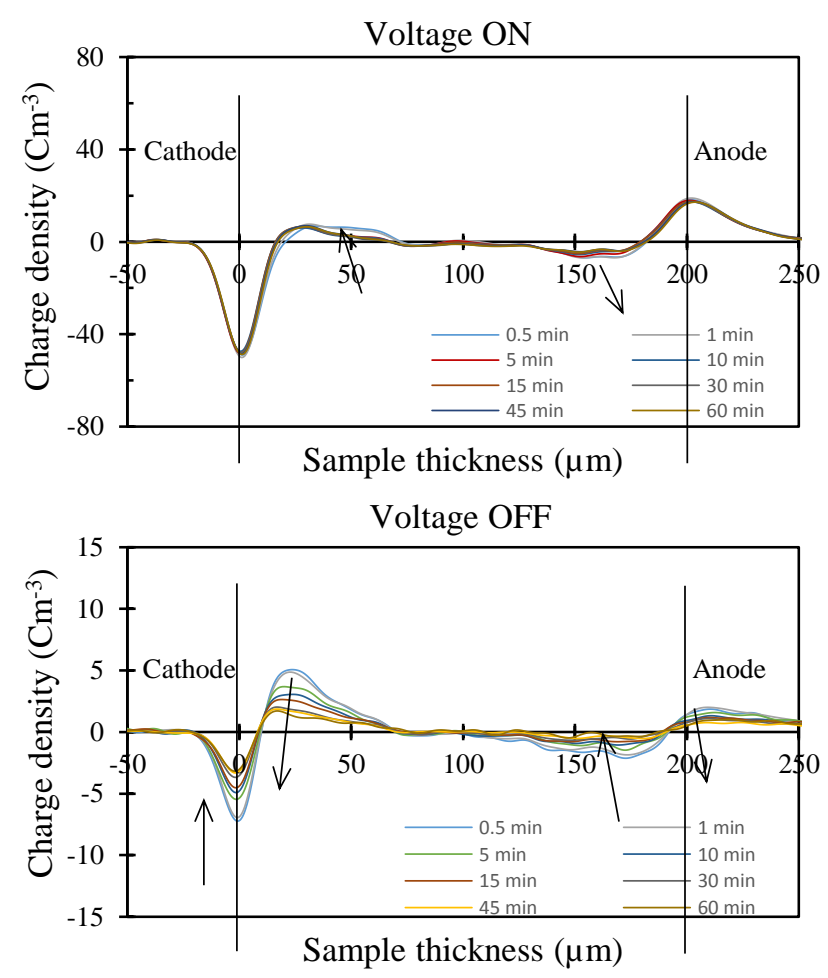

Fig. 3. PEA charge density profile of XLPE (un-degassed) thermally bonded Sc electrodes. The applied voltage was $40 \mathrm{kV} / \mathrm{mm}$ at room temperature. 
Fig. 3 shows the space charge evolution of undegassed XLPE sample. The formation of hetero-charge can be observed near both the cathode and the anode and the accumulation of hetero-charge is clearly shown in the depolarization data. This phenomenon is believed to be associated with the electric field assisted ionization process of the crosslinking byproducts and impurities within the material $[10,11]$. Fig. 4 shows the space charge behavior in the degassed samples after the voltage removal. It can be found that the large amount of positive charges present in the bulk of the sample. Hetero-charges are accumulated near the cathode, while homo-charges can be found near the anode.

The differences on the space charge behavior between the non-degassed samples and degassed samples can be studied by comparing the Fig.3 with Fig. 4, which clearly demonstrates that the space charge dynamics are significantly affected by the degassing process. After the degassing process, the main differences observed include 1. Homo-charges, instead of hetero-charges are accumulated near the anode; and 2. Large amount of charges (mostly positive charges) can be found in the sample bulk. Here, two possible explanations are proposed. Firstly, in a non-degassed sample, the high conductivity indicates that charges with large charge amount and/or high mobility propagate through the sample. This could result from the ionization of the by-products leading to the presence of charges with both polarities in the bulk. These charges are difficult to be measured by the PEA technique as only net charges can be detected. Therefore, only the large amount of hetero charges adjacent to the electrodes is observed in Fig. 3. On the other hand, in the degassed samples, some by-products are removed from the sample, leading to the significant decrease of the conductivity. In this work, the removed byproducts may contribute more in negative charges; therefore, the positive charges can be distinguished and observed in the bulk of degassed samples. The second possible explanation is the diffusion of the by-products or impurities from the bonded semicon layer into the bulk of the samples under high temperatures of the degassing process. The diffused byproducts or impurities could create deep traps to prevent charges propagating through the sample, resulting in the accumulation of charges in the bulk and the reduction of the conductivity. This diffusion may be dominated over the byproduct removal during the beginning period of the degassing process as well as with the extension of the degassing duration; the diffused by-products will be gradually removed from the bulk of the sample. However, further works are essential to validate these two possible explanations.

In order to investigate the effect of degassing duration, the space charge dynamics of the samples with 1-day degassing, 3day degassing and 6-day degassing are shown in Fig. 4 (A), (B) and $(\mathrm{C})$. It is evident that increasing the degassing time reduces the amount of accumulated charges in the system, especially the amount of negative charge. Indeed, only positive charge can be seen in the sample bulk. The majority of these positive charges are accumulated near the cathode, within the region from $10 \mu \mathrm{m}$ to $120 \mu \mathrm{m}$. It is also observed that the positive peaks in this region are gradually moving towards the cathode with the increase of the degassing duration. The maximum positive peak values and the total charge amount in the XLPE samples
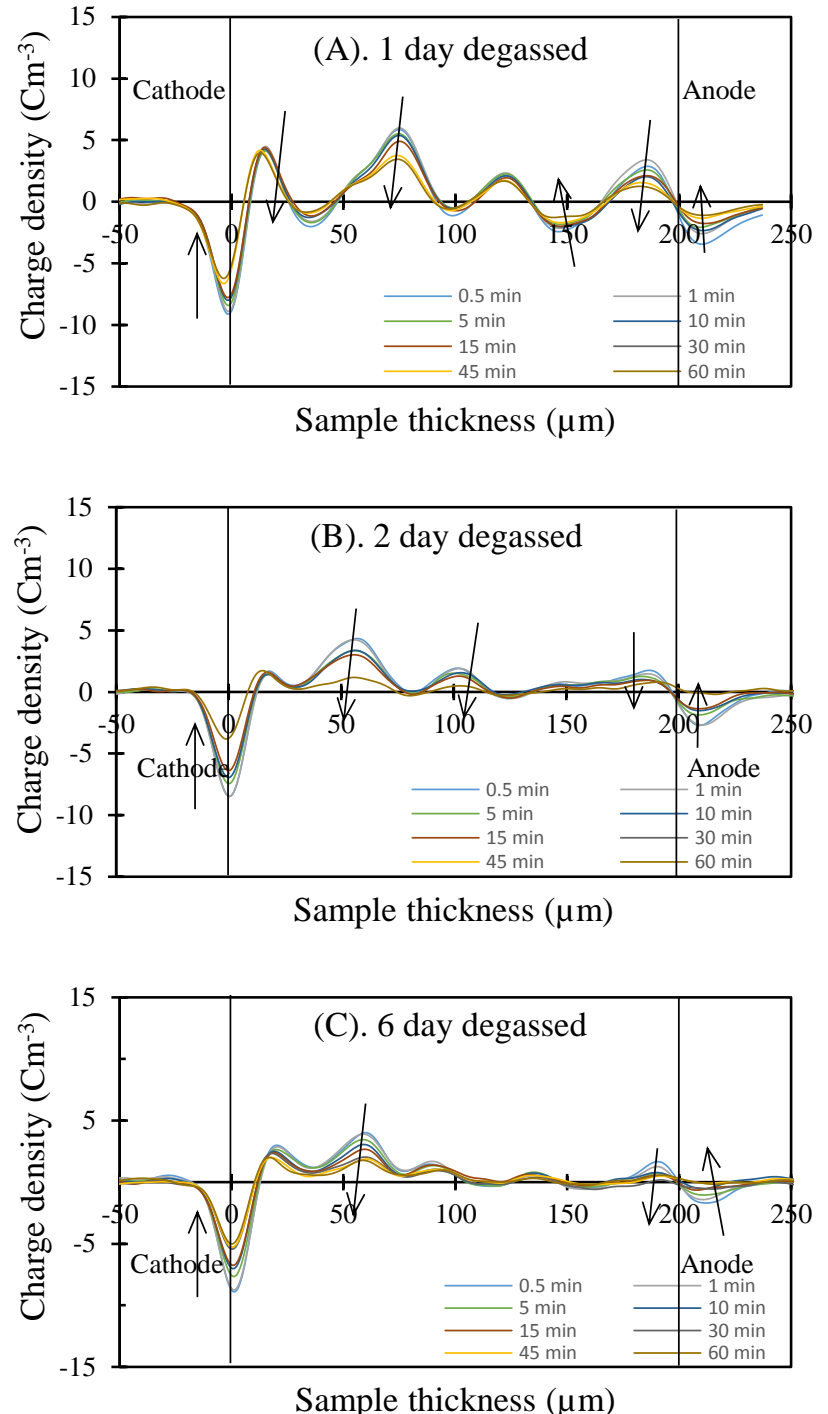

Fig. 4. PEA volts off space charge profile of XLPE samples thermally bonded Sc electrodes degassed at $80{ }^{\circ} \mathrm{C}$. The applied voltage for these samples was $40 \mathrm{kV} / \mathrm{mm}$ at room temperature.

obtained immediately after removal of the external voltage (i.e. at the start of charge decay) also summarized in Table I. Although the calculated absolute charge amount could be affected due to the unrecovered signal, the trend still can be clearly observed that both the maximum positive charge density and the absolute charge amount within the sample are decreased with increasing degassing time. However, a non-linear relationship between the degassing duration and the total charge can be found: for example, by comparing the charge amount in 1-day degassed sample with 3 and 6 days degassed sample, the charge amount decreases by $30 \%$ and $34 \%$, respectively.

TABLE I. SUMMARY OF CHARGE AMOUNT AND MAXIMUM PEAK VALUE VS. DEGASSING DURATION.

\begin{tabular}{|c|c|c|}
\hline $\begin{array}{c}\text { Degassing duration } \\
\text { (day) }\end{array}$ & $\begin{array}{c}\text { Maximum peak } \\
\text { value }\left(\mathbf{C} / \mathbf{m}^{\mathbf{3}}\right)\end{array}$ & $\begin{array}{c}\text { Total charge amount } \\
(\mathbf{n C})\end{array}$ \\
\hline 1 & 6.1 & 41.3 \\
\hline
\end{tabular}




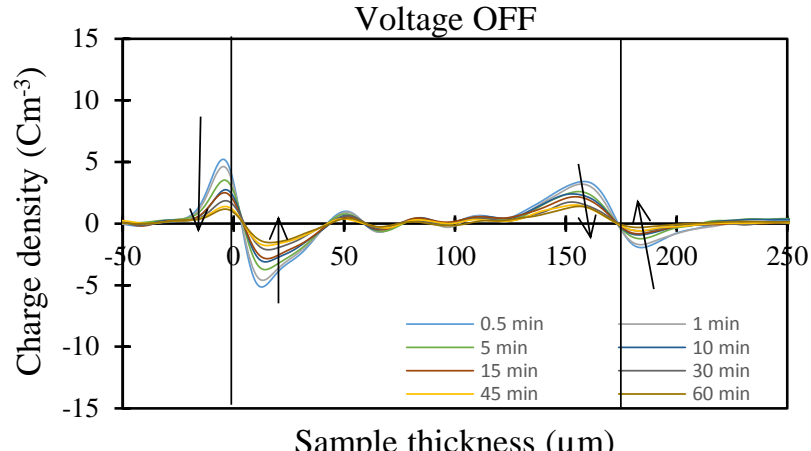

Sample thickness $(\mu \mathrm{m})$

Fig. 5. PEA space charge profile of XLPE (degassed) thermally bonded HVDC grade Sc electrodes. The applied voltage was $40 \mathrm{kV} / \mathrm{mm}$ at room temperature.

\begin{tabular}{|c|c|c|}
\hline 3 & 4.6 & 28.6 \\
\hline 6 & 3.9 & 27.2 \\
\hline
\end{tabular}

In addition, the space charge charactersitics in such bonded samples are greatly dependant on the characteristics of the

Cathode

Anode

semiconductive materal. To validate this, XLPE sample bonded with a HVDC cable grade semicon was undertaken and the results are shown in Fig. 5. Interestingly, the formation of homo-charge at the both electrodes is clearly visible, and such difference indicates the charge injection and extraction is dominant over ionic dissociation. For instance, the bulk samples of Fig. 4 and Fig. 5 are produced from the same material, however, by replacing the semiconductive material triggered the outcomes. This strongly suggests that the electrodes also play a significant role in the charge injection and the nature of the semiconducting material has an important role in space charge accumulation in the XLPE bulk, affecting injection and extraction of charges.

\section{CONCLUSIONS}

In this paper, the influence of degassing on space charge and DC conductivity of XLPE bulk thermally bonded with HVDC cable grade semiconductive material is reported. The results clearly suggests the space charge dynamics are significantly affected by the degassing process, such as, homo-charge distribution is dominant in the degassed XLPE sample, whereas, hetero-charge is dominant in undegassed samples. This effect could be attributed to the charge injection from the electrodes in the degassed sample and residual byproducts of the crosslinking reaction in undegassed sample. This phenomenon was further confirmed from conduction tests, in which undegassed samples have shown much higher conduction. Diffusion of the by-products or impurities from the bonded semicon layer into the bulk under high temperature condition could also have a role in the accumulation of charges such as creating deep traps to prevent charges propagating through the sample resulting in the accumulation of charges in the bulk. However, further works are essential to validate this phenomenon. The effects on degassing duration investigated in the current studies revealed that both the maximum positive charge density and the absolute charge amount within the sample are decrease with increasing degassing time, thereby longer degassing duration is favorable for the XLPE as long as space charge is concern. Moreover, experimental results shows that the nature of semiconducting material has an important role in the space charge accumulation affecting injection and extraction of charges in the bulk XLPE sample. Overall, this can be conclude that the nature of space charge, such as, hetero and homo-charge depends strongly on the byproducts in the XLPE material and the type of semiconductive material. Findings of this work could provide fundamental information on the degassing process and the selection of right semiconductive/insulation combination.

\section{ACKNOWLEDGMENT}

The authors are grateful for the financial support from the State Grid Cooperation of China: Research on Key Technologies of Insulation Material and Accessories for $320 \mathrm{kV}$ HVDC XLPE Cable System (SGRIZLJS(2014)888).

\section{REFERENCES}

[1] Y. L. Chong, G. Chen, and Y. F. F. Ho, "The effect of degassing on morphology and space charge," in Solid Dielectrics, 2004. ICSD 2004. Proceedings of the 2004 IEEE International Conference on, 2004, pp. 162-165 Vol.1.

[2] S. Annika, B. Jan-Ove, W. Detlef, and P. Rudi, "Comparison Of Different Analytical Test Methods To Monitor Crosslinking By-Products In XLPE Insulated Cables," in 7th International Conference on Power Insulated Cables (JICABLE-07), Paris, 2007.

[3] S. H. Lee, J. K. Park, J. H. Han, and K. S. Suh, "Space charge and electrical conduction in maleic anhydride-grafted polyethylene," IEEE Transactions on Dielectrics and Electrical Insulation, vol. 2, pp. 11321139, 1995

[4] L. H. U. Andersson and T. Hjertberg, "The effect of different structure parameters on the crosslinking behaviour and network performance of LDPE," Polymer, vol. 47, pp. 200-210, 2006.

[5] M. Fu, L. A. Dissado, G. Chen, and J. C. Fothergill, "Space charge formation and its modified electric field under applied voltage reversal and temperature gradient in XLPE cable," IEEE Transactions on Dielectrics and Electrical Insulation, vol. 15, pp. 851-860, 2008.

[6] W. Choo, G. Chen, and S. G. Swingler, "Electric field in polymeric cable due to space charge accumulation under DC and temperature gradient," IEEE Transactions on Dielectrics and Electrical Insulation, vol. 18, pp. 596-606, 2011.

[7] Y. L. Chong, G. Chen, I. L. Hosier, A. S. Vaughan, and Y. F. F. Ho, "Heat treatment of cross-linked polyethylene and its effect on morphology and space charge evolution," IEEE Transactions on Dielectrics and Electrical Insulation, vol. 12, pp. 1209-1221, 2005.

[8] N. Hirai, Y. Maeno, T. Tanaka, Y. Ohki, M. Okashita, and T. Maeno, "Effect of crosslinking on space charge formation in crosslinked polyethylene," in Properties and Applications of Dielectric Materials, 2003. Proceedings of the 7th International Conference on, 2003, pp. 917920 vol.3.

[9] W. S. Lau and G. Chen, "Simultaneous Space Charge and Conduction Current Measurements in Solid Dielectrics under High DC Electric Field," in Proceedings in International Conference on Condition Monitoring and Diagnosis, Korea, 2006, pp. 1-5.

[10] Y. F. F. Ho, H. G. Chen, A. E. Davies, S. G. Swingler, S. J. Sutton, and R. N. Hampton, "Effect of semiconducting screen on the space charge dynamic in XLPE and polyolefin insulation under DC and $50 \mathrm{~Hz} \mathrm{AC}$ electric stresses conditions," IEEE Transactions on Dielectrics and Electrical Insulation, vol. 10, pp. 393-403, 2003.

[11] Y. Li and T. Takada, "Experimental observation of charge transport and injection in XLPE at polarity reversal," Journal of Physics D: Applied Physics, vol. 25, p. 704, 1992. 